\title{
Probiotics in infectious diarrhoea in children: are they indicated?
}

\author{
Y. Vandenplas • S. Salvatore $\cdot$ M. Vieira $\cdot$ T. Devreker • \\ B. Hauser
}

Published online: 9 October 2007

(C) Springer-Verlag 2007

An incorrect spelling of the third author's name was supplied.

The correct name is: M. Vieira

The online version of the original article can be found at http://dx.doi. org/10.1007/s00431-007-0497-9.

Y. Vandenplas $(\bowtie) \cdot$ T. Devreker $\cdot$ B. Hauser

Universitair Ziekenhuis Brussel Kinderen,

Vrije Universiteit Brussel,

Laarbeeklaan 101,

1090 Brussels, Belgium

e-mail: yvanvandenplas@az.vub.ac.be

S. Salvatore

Clinica Pediatrica di Varese, Università dell'Insubria,

Varese, Italy

M. Vieira

Hospital Pequeno Príncipe,

Curitiba, Brazil 